\title{
An Evaluation of IPv6 Multicast Router in JGN IPv6 Networks
}

\author{
Yukiji Mikamo \\ TAO Okayama Interoperability \\ \& Evaluation Laboratory \\ Ouchida 675, Teleport Okayama \\ Okayama-shi, Okayama, 701-0165 Japan \\ Hisayoshi Hayashi \\ Hitachi, Ltd., IP Network Division \\ Horiyamashita 1, Hadano-shi \\ Kanagawa, 259-1392 Japan
}

Kazumasa Kobayashi

Kurashiki University of Science and the Arts

Nishinoura 2640, Tsurajima-cho

Kurashiki-shi, Okayama, 712-8505 Japan

\author{
Satoshi Katsuno \\ TAO Tokyo Research \& Operation Center \\ Otemachi 1-8-1 KDDI Otemachi Bldg. \\ Chiyoda-ku, Tokyo, 100-0004 Japan
}

Takashi Miyake

Kurashiki University of Science and the Arts

Nishinoura 2640, Tsurajima-cho

Kurashiki-shi, Okayama, 712-8505 Japan

\author{
Hiroshi Esaki \\ The University of Tokyo \\ Hongo 7-3-1 Bunkyo-ku \\ Tokyo, 113-8656 Japan
}

\begin{abstract}
This paper describes an evaluation of IPv6 multicast routers. The JGN IPv6 project has performed various evaluations and verifications of IPv6 routers. Seven (7) types of routers from six vendors were tested, for which PIM-SM was employed as an IPv6 multicast protocol for the evaluation. The evaluated items include PIM-SM interoperability, as well as detour, RP and BSR behavior. Several tests were performed on ATM and Ethernet connections. Detailed results of these verifications are shown in this report. No fatal problem were found and only a few minor issues occurred while conducting these evaluations.
\end{abstract}

\section{Introduction}

Multicasting is becoming more necessary and important in broadband environments. During IPv4, the multicast was implemented in many routers, yet it was not widely used due mainly to the implementation of IGMP snooping function in connected switches, problems in network bandwidth used, as well as insufficient performance in multicast packet transaction of the routers themselves. Thus, its interoperability was not criticized.

In current IPv6 environments, the bandwidth problem does not exist, while problems in transaction efficiency were solved by improving performance by using a hardware-based transaction circuit. Thus led ultimately to successes in multicast.

To verify its interoperability means that multicast can be realized from among different network organizations, while new services, which appear, are expected to act as part of the social infrastructure in the future.

\section{Research Details}

\subsection{Router's IPv6 compatibility}

The Telecommunication Advancement Organization of Japan (TAO) [8] has evaluated IPv6 [3] routers on their performance and functionality based on their interoperability. The targets of the evaluation are focused on basic communication, performance, unicast routing protocols and mandatory functions as in IPv4.

At present, the verification of OSPFv3 [2] routing protocol has been completed, and construction of a backbone network using routers possessed by the JGNv6 [6] project can be realized. The deployment of OSPFv3 in JGNv6 network is coming to fruition.

\section{IPv6 Multicast Implementation Status}

\subsection{Evaluation Data}

The evaluated items are as follows: 


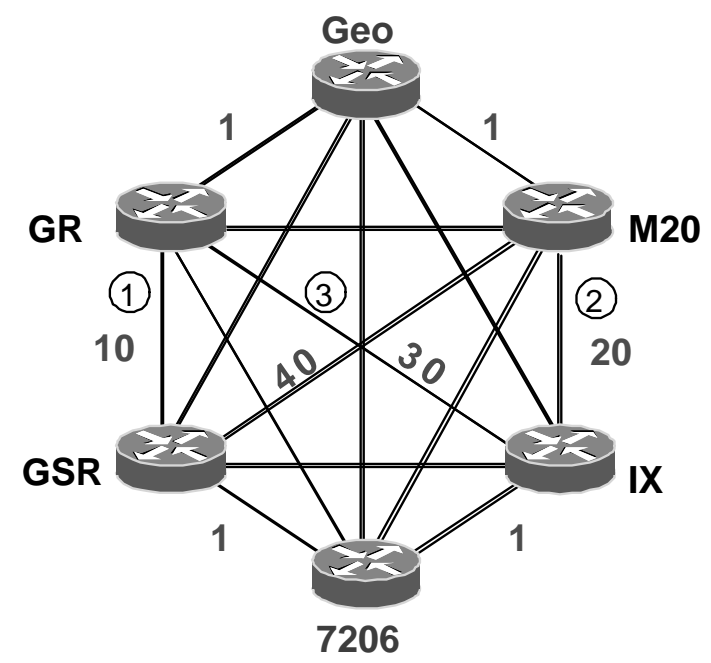

Figure 1. Example of OSPFv3 Evaluation Network Structure.
(1) Unicast Routing: OSPFv3
(2) Multicast Group Management: MLD [4]
(3) Multicast Routing: PIM-SM [5]
(4) Multicast Group Address: ff18::xy

The reasons why PIM-SM was selected in the evaluation of IPv6 multicast were that there was no other choice when scalability in large-scale networks were considered, while PIM-SM was implemented by router vendors ahead of other protocols. This protocol is standard in current IPv6 multicast.

Despite this, some vendors only implement PIMSSM [1], which is an extension of PIM-SM. A specific example is Juniper's M20 router. Yet it does not support unicast tunnel function between the DR (Designated Router) and RP (Rendezvous Point) in PIM-SM. It works when the router is neither a RP nor source DR, thus the evaluation was performed under this condition. The versions of router software that were employed in the evaluation are shown in the following table:

\begin{tabular}{|l|l|l|}
\hline \multicolumn{1}{|c|}{ Vendor } & \multicolumn{1}{|c|}{ Name } & \multicolumn{1}{c|}{ Router OS } \\
\hline $\begin{array}{l}\text { Cisco } \\
\text { Systems }\end{array}$ & GSR12406 & IOS 12.0(26)S Patch Ver. \\
\cline { 2 - 3 } & $\begin{array}{l}\text { 7206VXR } \\
\text { (NPE3000) }\end{array}$ & IOS 12.3(2) \\
\hline $\begin{array}{l}\text { Juniper } \\
\text { Networks }\end{array}$ & M20 & JUNOS6.0 R1.5 \\
\hline HITACHI & GR2000-6H & S-9181-61 07-03-/C \\
\hline NEC & IX5030 & 7.6.20 \\
\hline FUJITSU & $\begin{array}{l}\text { GeoStream } \\
\text { R920 }\end{array}$ & E10V03L50C09 \\
\hline Furukawa & $\begin{array}{l}\text { FITELnet- } \\
\text { G20 }\end{array}$ & v01.32 \\
\hline
\end{tabular}

\subsection{Implementation status}

The evaluated routers are possessed by TAO, or introduced by router vendors. Some of the routers introduced in JGNv6 did not support IPv6 multicast. In thses instances, the router's successors were employed for the evaluation. The status of implementation is as shown in the following table:

\begin{tabular}{|l|l|c|c|}
\hline \multicolumn{1}{|c|}{ Vendor } & \multicolumn{1}{|c|}{ Name } & Static-RP & BSR \\
\hline $\begin{array}{l}\text { Cisco } \\
\text { Systems }\end{array}$ & GSR12406 & $\bigcirc$ & $\begin{array}{c}\triangle \\
\text { Special IOS }\end{array}$ \\
\cline { 2 - 4 } & 7206VXR & $\bigcirc$ & $\begin{array}{c}\triangle \\
\text { Special IOS }\end{array}$ \\
\hline $\begin{array}{l}\text { Juniper } \\
\text { Networks }\end{array}$ & M20 & $\bigcirc$ & $\begin{array}{c}\times \\
\text { PIM-SSM }\end{array}$ \\
\hline Hitachi & GR2000-6H & $\times$ & $\bigcirc$ \\
\hline NEC & IX5030 & $\bigcirc$ & $\bigcirc$ \\
\hline Fujitsu & GeoStream R920 & $\bigcirc$ & $\bigcirc$ \\
\hline Furukawa & FITELnet-G20 & $\bigcirc$ & $\bigcirc$ \\
\hline
\end{tabular}

\subsection{Evaluated items}

The following items were selected as mandatory functions for IPv6 multicast evaluated items:

(1) Basic evaluation of PIM-SM interoperability

(2) PIM-SM / OSPFv3 detour function behavior

(3) Evaluation of RP behavior

(4) Evaluation of BSR behavior

Items (1) to (3) were evaluated in ATM point-to-point connection topology (Figure 2), while item (4) was evaluated in an Ethernet broadcast environment (Figure 3) in considering actual operations on JGNv6 networks. Furukawa's FITELnet-G20, which does not have an ATM interface, was connected via GSR12406 in ATM point-to-point connection topology.

\section{Results of Interoperability Evaluation}

\subsection{Evaluation in ATM connection (1) - (3)}

\subsubsection{Connection topology of basic evaluation in PIM- SM interoperability}

Firstly, basic connections in PIM-SM were evaluated before the evaluation of each function. The reason to perform this evaluation is that there were no known examinations of PIM-SM evaluation with so many types of routers such as those used in this evaluation, while the software brought by vendors included special versions.

The specific connection topology is shown in Figure 4. Interoperability of all routers can be evaluated in the simplest fashion, by employing GR2000-6H, as described in 

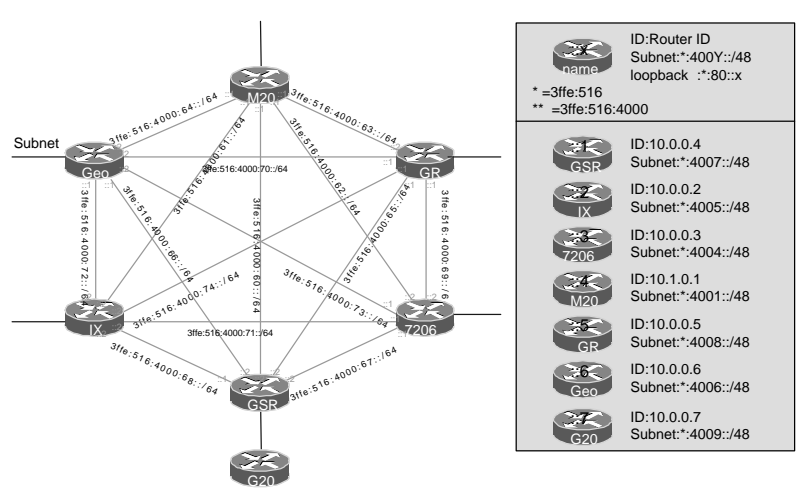

Figure 2. Network Topology of the Evaluation in ATM Connection
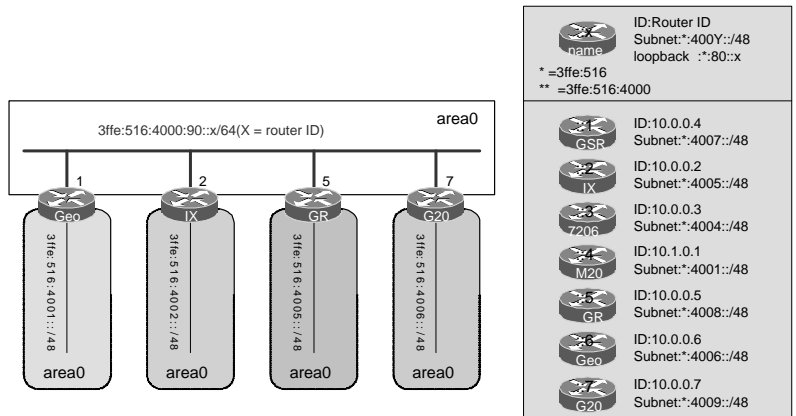

Figure 3. Network Topology of the Evaluation in Ethernet Connection

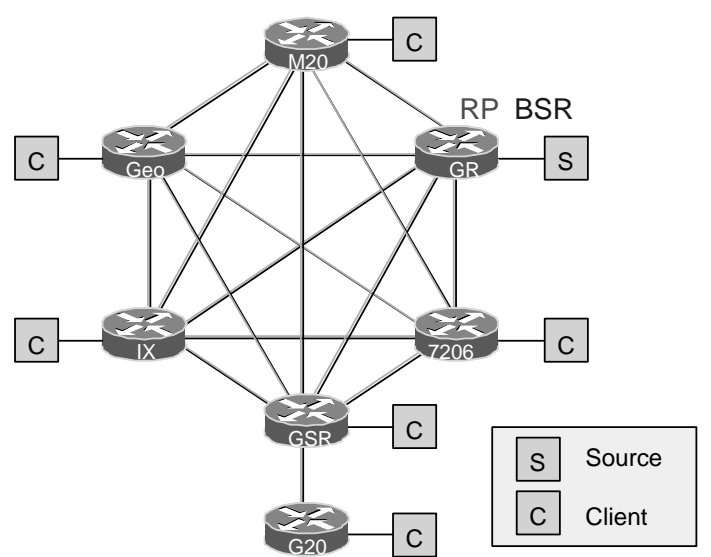

Figure 4. Connection Topology of Basic Evaluation the implementation status section, which cannot be a static $\mathrm{RP}$, as RP, BSR, and source.

DVcommXP by Fatware and DVTS by WIDE project were employed in all evaluations.

4.1.1.1 PIM-Hello Option Compatibility Point-to-point addresses of ATM interfaces are originally link-local addresses. However, in this evaluation, global addresses were assigned, while PIM-Hello option compatibility and its behavior were confirmed [7]. Though it was found that M20 did not perform the PIM Hello option, it ignored the option and no problem was discovered in its communication.

Aug 28 16:17:38 PIM Hello Unknown option: 65001 $16: 24: 25.503754$ Out fe80::2a0:a5ff:fe3d:1b42> ff02::d: pim v2 Hello (Hold-time 1m45s) (OLD-DRPriority: 1) [class 0xc0] [hliml]

PIM-Hello does not have any option in M20.

Aug 28 16:25:28 PIM at-0/0/0.61 SENT 3ffe:516:40 00:61::1 -> ff02::d V2 Hello hold 105 T-bit LAN prune $500 \mathrm{~ms}$ override $2000 \mathrm{~ms}$ pri 1 sum $0 \times 957 \mathrm{f}$ len 26

4.1.1.2 Evaluation results of basic interoperability Received messages in multicast clients under each router were confirmed. No problem were discovered in basic interoperability.

\subsubsection{PIM-SM / OSPFv3 detour function behavior}

One of the important PIM-SIM functions is multicast packet communication via the shortest path. This function was evaluated by detouring unicast route by OSPFv3, while IPv6 backbone was assumed.

ATM links were interrupted in numerical order, as shown in Figure 5. The router in which the route change occurred was confirmed to have received packets in the shortest path. To change routes immediately when interfaces go down, its logical interfaces were interrupted in software methods together with an opposite side router simultaneously.

4.1.2.1 Evaluation results of detour function The time between the selection of the shortest path and receipt of packets are as shown in Table 1.

Except for GeoStream, when each router received "Join" messages from the lower reaches, rerouting was performed and they were replicated into required interfaces. It was 
Table 1. Selection of Shortest Path and Receipt of Packets

\begin{tabular}{|l|c|c|c|c|c|c|c|l|}
\hline & M20 & GR & 7200 & GSR & G20 & IX & Geo & Notes \\
\hline GR-GEO shut down & - & - & - & - & - & - & 120 over & GEO's specification for MLD \\
\hline GR-IX shut down & - & - & - & - & - & 8 & - & \\
\hline GR-GSR shut down & - & - & - & 11 & - & - & - & \\
\hline GR-7200 shut down & - & - & 7 & - & - & - & - & \\
\hline IX-M20 shut down & - & - & - & - & - & 32 & & \\
\hline M20-7200 shut down & - & - & 7 & - & - & - & - & \\
\hline M20-GSR shut down & - & - & - & 9 & - & - & - & \\
\hline Geo-7200 shut down & - & - & 155 & - & - & - & - & \\
\hline IX-7200 shut down & - & - & $\times$ & - & - & - & - & \\
\hline Geo-GSR shut down & - & - & - & 8 & - & - & - & \\
\hline
\end{tabular}

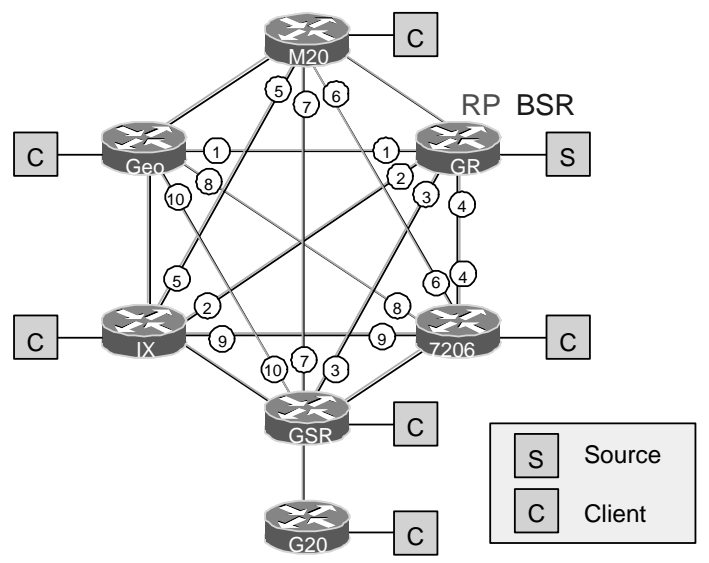

Figure 5. Connection Topology of Basic Interoperability Evaluation

found that GeoStream waited query interval time before changing its PIM-Join target of in the specification.

4.1.2.2 Evaluation items in detour function There are two items to be considered. The first is that GeoStream takes a long time to redistribute packets as it suppresses its load when encapsulation is performed. Yet, other routers does not show such phenomenon thus the problem was with GeoStream. This phenomenon was indicated to the router vendor that they were asked to consider it as such.

The second item is that when the link 9 in Figure 5 was interrupted, IX did not receive assumed packets, which were replicated from GSR. This appears to be the following phenomenon between 7206 and IX:

- 7206:PIM Register Stop $\rightarrow$ IX:does not receive messages

However, it worked without any problems as GSR and 7206, GSR did not find PIM-Join from IX between GSR

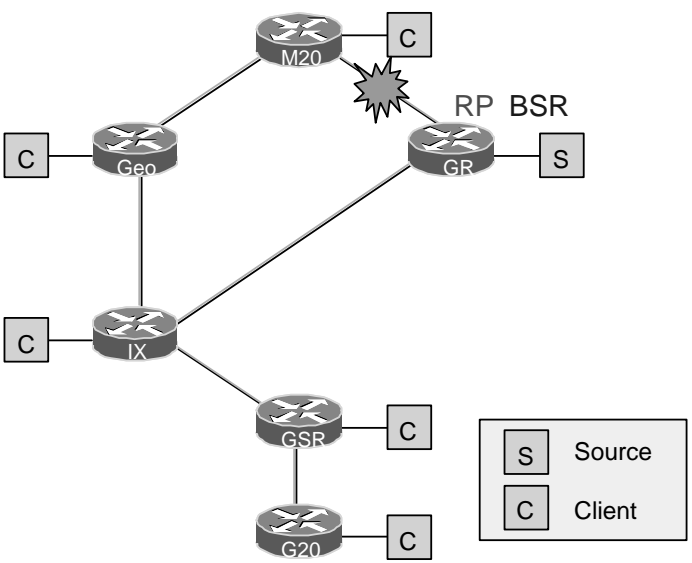

Figure 6. Connection Topology when GSR and 7206 Were Eliminated

and IX, while the same phenomenon was found to occur. To solve this problem, connection topology was changed and GSR and 7206 were eliminated (Figure 6), and it was confirmed to work between IX and GeoStream. The problem was found between GSR and 7206.

The problem in special router software with BSR function was found, though contact with the vendors.

When router software in GSR and 7206 was changed to a previous one, no problem was found. Then, the evaluation

Table 2. Results when GSR and 7206 Were Eliminated.

\begin{tabular}{|l|c|c|c|c|c|}
\hline & M20 & GR & G20 & IX & Geo \\
\hline GR-M20 shut down & 4 & - & - & 5 & - \\
\hline GR-M20 no shut down & 0 & - & - & 0 & 0 \\
\hline
\end{tabular}




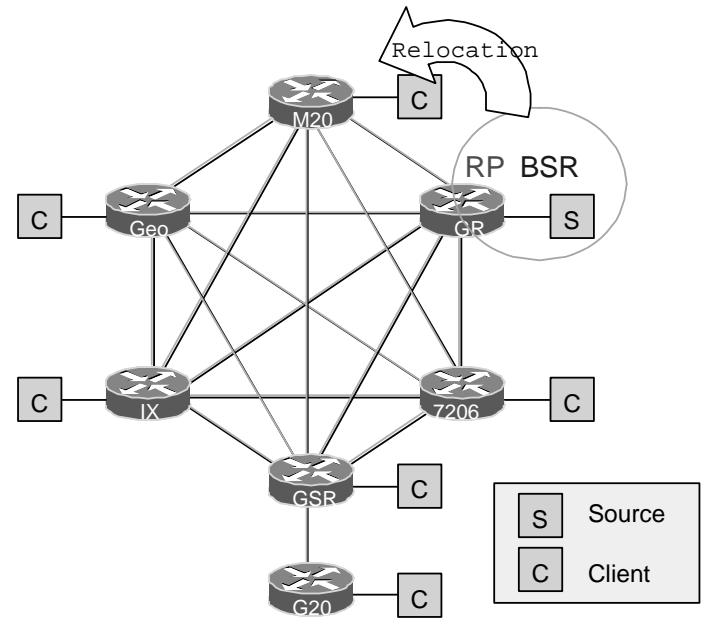

Figure 7. Evaluation of RP Function

was continued with the software without the BSR function.

\subsubsection{Evaluation of RP behavior}

Each router's behavior as RP was evaluated. For M20, which performs PIM-SM, as described above, the evaluations were performed where RP and DR, being neither RP nor source RP.

The evaluation process is shown in Figure 7. RP, BSR and Source were relocated under each router, while instances where RP is not source were also evaluated. Though this is not an ideal environment, such situations often occur in actual networks.

4.1.3.1 Evaluation results of $R P$ behavior The results of the evaluation are shown in Table 3.

As shown in Table 3, when IX was RP and source was under GSR or 7206, replication did not work. The reason for this phenomenon was found to be that IX submitted "Register-Stop" without (S,G) and GSR did not send packets. It was found that the problem depended on how the vendor implemented this, thus the vendor was requested to make improvements.

\subsection{Evaluation in Ethernet connection(4)}

\subsubsection{Evaluation topology of BSR function}

The BSR function was evaluated for GeoStream, IX, GR and G20, which implemented this function. By evaluating each router's match concerning RP and BSR, BSR problems that occur due to matching each router were clarified.

Each group source was added under each router. As a result, four multicast groups existed in the network, which BSR and RP handled.

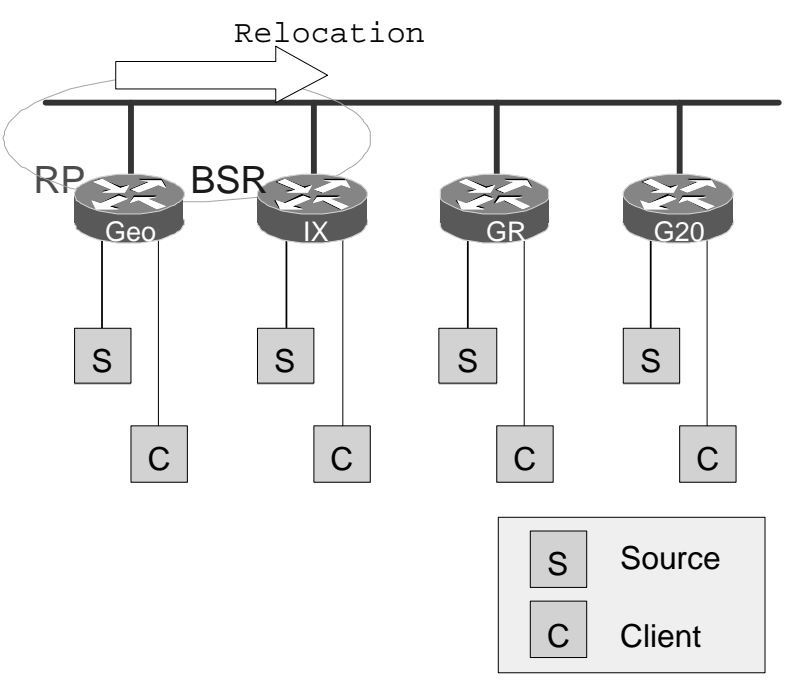

Figure 8. BSR Function Verification

BSR and RP were switched by managing their priorities. For BSR, a larger value takes high priority, while a smaller value takes high priority for RP candidates. However, GeoStream cannot set RP priorities at present so its loopback address was set to the smallest value.

\subsubsection{Evaluation results of BSR function and problems} Evaluation results of all matches are shown in Table 4.

As shown in this table, GR were rarely evaluated. Problems were found when the source was placed under other routers. For this topology, GR can create correct $(G, R)$ when GR was RP, BSR itself, and the source was under it, yet, when the source is under other routers, it cannot send $(G, R)$ properly. This phenomenon occerred only when multiple PIM routers and multiple sources existed in a single broadcast network. Vendors were asked to investigate this phenomenon.

\section{Discussion}

As some routers do not have RP and BSR functions, an overall summary cannot be presented in terms of IPv6 PIMSM interoperability. However, concerning routers that implemented the functions, very few problems were located. At the practical operation level, there is sufficient performance for use by locating the RP, BSR and source in the proper locations. This can be used more efficiently if multicast applications that use smaller bandwidths are employed. This evaluation was affected by bandwidth problems due to an over $30 \mathrm{Mbit} / \mathrm{s}$ DV stream. No problems should exist when all backbone routers are connected to high-speed circuits. However, if one of the routers is connected via a low speed circuit, it is sometimes flooded by relays from route 
Table 3. Evaluation Results of RP Behavior

\begin{tabular}{|c|c|c|c|c|c|c|c|c|c|}
\hline RP & Source & M20 & GR & 7200 & GSR & $\mathrm{G} 20$ & IX & Geo & Notes \\
\hline M20 & M20 & 1 & 1 & 1 & 1 & 1 & 1 & 1 & \\
\hline M20 & $\bar{x}$ & - & - & - & - & - & - & - & M20 doesn't support RP $\neq$ source \\
\hline Geo & Geo & 3 & 3 & 3 & 3 & 3 & 3 & 3 & $\begin{array}{l}\text { As a specification for joining simultaneously, it waits } \\
\text { a certain period }\end{array}$ \\
\hline Geo & IX & 4 & 4 & 4 & 4 & 5 & 4 & 4 & Bit rate of DVTS is limited to $20 \mathrm{Mbit} / \mathrm{s}$ due to $\mathrm{OC} / 3$ \\
\hline IX & IX & 1 & 1 & 1 & 1 & 1 & 1 & 1 & Bit rate of DVTS is limited to $20 \mathrm{Mbit} / \mathrm{s}$ due to $\mathrm{OC} / 3$ \\
\hline IX & GSR & - & - & - & - & - & - & - & $\begin{array}{l}\text { The first test did not work. This phenomenon was } \\
\text { confirmed twice. This was not due to the interop- } \\
\text { erability of PIM-SM, but that the NEC IX submits } \\
\text { register-stop without creating }(\mathrm{S}, \mathrm{G}) \text {. }\end{array}$ \\
\hline Geo & GSR & - & - & - & - & - & - & - & $\begin{array}{l}\text { The problem did not occurr in the second test. Packet } \\
\text { flow was shown to be normal. The vendor said it } \\
\text { might be due to an internal problem in GEO when } \\
\text { it was evaluated. }\end{array}$ \\
\hline GSR & GSR & 1 & 1 & 1 & 1 & 1 & 1 & 1 & \\
\hline GSR & G20 & 1 & 1 & 1 & 1 & 1 & 1 & 1 & \\
\hline G20 & G20 & 1 & 1 & 1 & 1 & 1 & 1 & 1 & \\
\hline G20 & 7200 & 1 & 1 & 1 & 1 & 1 & 1 & 1 & Bit rate of DVTS is limited to $20 \mathrm{Mbit} / \mathrm{s}$ due to $\mathrm{OC} / 3$ \\
\hline 7200 & 7200 & 1 & 1 & 1 & 1 & 1 & 1 & 1 & Bit rate of DVTS is limited to $20 \mathrm{Mbit} / \mathrm{s}$ due to $\mathrm{OC} / 3$ \\
\hline 7200 & Geo & 1 & 1 & 1 & 1 & 1 & 1 & 1 & $\begin{array}{l}\text { Each router performed under } 2000 \text { pps. Block noise } \\
\text { occurred because the bandwidth usage was } 22 \mathrm{Mbit} / \mathrm{s} \text {. }\end{array}$ \\
\hline IX & 7200 & - & - & - & - & - & - & - & $\begin{array}{l}\text { The same problem as IX-GSR, Geo-GSR occurred. } \\
\text { Routers except for local } 7200 \text { could not connect. }\end{array}$ \\
\hline GSR & 7200 & \multicolumn{7}{|c|}{ Not measured } & \\
\hline
\end{tabular}

Table 4. Results of BSR Evaluation

\begin{tabular}{|c|c|c|c|c|}
\hline $\mathrm{BSR} \quad \mathrm{RP}$ & GR & IX & Geo & G20 \\
\hline$\overline{\overline{\text { GR }}}$ & $\overline{0}$ & $\overline{-1}$ & $\overline{-1}$ & $\bar{~}$ \\
\hline IX & - & 0 & 0 & 0 \\
\hline Geo & - & 0 & $\bigcirc$ & 0 \\
\hline G20 & - & 0 & 0 & 0 \\
\hline
\end{tabular}

changes. In this evaluation, $155 \mathrm{Mbit} / \mathrm{s}$ ATM circuits were flooded. This fact should be considered when actual networks are designed.

The evaluation results are fed back to each vendor. Router software version upgrades were requested to solve each problem.

\section{Conclusion}

One issue which caused concern was performance. For unicast performance, no problem was located in the various evaluation tests for target routers. However, some routers were expected to have performance problems in IPv6 multicast transactions. In practice, software-based relays were limited to temporary cases, when no route table was found, or where there was an encapsulated relay to RP. Hardware-based transactions are primarily employed, thus performance issues may be disappear over time.

However, the routers in JGNv6 project might be solved by making requests to vendors on performance problems. Consideration is needed when other routers are employed. In conclusion, the necessity of interoperability evaluations of different routers is realized. The evaluation of interoperability cannot be performed entirely in the vendor's laboratory alone. For example, some problems were discovered when a vendor brought a draft code to implement a new function, and their new codes were tested in their internal environment. The problem were revealed only during particular interfaces. Such problems are hard to find in their laboratory alone because some interfaces are normally unavailable there.

In the future, IPv6 multicast will be deployed in JGN IPv6 networks. Evaluations of multicast will be performed in real operating environments. In tested environments, no unicast traffic coexisted, thus unpredictable phenomenon might not occur. During IPv4, multicast was used in isolated networks; however, it will work as an infrastructure suitable to a ubiquitous society during IPv6. 


\section{Acknowledgements}

We sincerely thank the persons who related with this JGN IPv6 project in Telecommunications Advancement Organizations of Japan (TAO). Also, we thanks to the Ministry of Public Management, Home Affairs, Posts and Telecommunications, who gave us the opportunity to be involved in the construction of the JGN IPv6 network. We would also like to take this opportunity to thank the network engineers who took part in the operation of the JGN IPv6 network for their cooperation.

\section{References}

[1] B. Cain and H. Holbrook. Source-specific multicast for IP. IETF draft-ietf-ssm-arch-03.txt, May 2003.

[2] R. Coltun, D. Ferguson, and J. Moy. OSPF for IPv6. IETF RFC 2470, Dec. 1999.

[3] S. Deering and R. Hinden. Internet protocol version 6(IPv6) specification. IETF RFC 2460, Dec. 1999.

[4] S. E. Deering, W. C. Fenner, and B. Haberman. Multicast listner discovery (MLD) for IPv6. IETF RFC 2710, Oct. 1999.

[5] B. Fenner, M. Handley, B. Holbrook, and I. Kouvelas. Protocol indepemdent multicast - sparse mode (PIM-SM): Protocol specification (revised). IETF draft-ietf-pim-sm-v2-new-07.txt, Mar. 2003.

[6] K. Kobayashi, S. Katsuno, K. Nakamura, Y. Mikamo, H. Hayashi, A. Machizawa, and H. Esaki. JGN IPv6 network. In Proceedings of The 2003 International Symposium on Applications and the Internet (SAINT) Workshop, Orlando, FL, Jan.27-31 2003.

[7] S. Suzuki and T. Jinmei. PIM upstream detection among multiple addresses. IETF draft-suz-pim-upstream-detection00.txt, Feb. 2003.

[8] TAO. Japan Gigabit Network home page. http://www.jgn.tao.go.jp/. 